\title{
High prevalence of polycystic ovary syndrome in women with mild hirsutism and no other significant clinical symptoms
}

\author{
Gaetana Di Fede, M.D., Pasquale Mansueto, M.D., Ilenia Pepe, M.D., Giovam Battista Rini, M.D., \\ and Enrico Carmina, M.D. \\ Department of Clinical Medicine, University of Palermo, Palermo, Italy
}

\begin{abstract}
Objective: To verify the conclusions of the Endocrine Society Guidelines that patients with mild hirsutism and no other important clinical signs (menstrual irregularities, infertility, central obesity, acanthosis nigricans, rapid progression of the hirsutism, clitoromegaly) should not be further studied.

Design: Retrospective study in patients referred because of mild hirsutism and no other clinical signs.

Setting: Department of Clinical Medicine of the University of Palermo.

Patient(s): One hundred fifty-two patients with mild hirsutism.

Intervention(s): Measurement of serum testosterone, dehydroepiandrosterone sulfate, 17-OH-Progesterone, assessment of ovulation by measurement of progesterone in 21 to 24 days and ovarian ultrasound.

Result(s): In 72 (47\%) patients a diagnosis of polycyctic ovarian syndrome (PCOS) was performed. Polycyctic ovarian syndrome patients included 56 patients with the mild ovulatory form (OV-PCOS) but also 16 patients with the anovulatory form (classic PCOS). Three (2\%) patients had nonclassic adrenal hyperplasia.

Conclusion(s): Because of the high prevalence of PCOS and the possibility of finding nonclassic 21-hydroxylase deficiency, patients with mild hirsutism need a diagnostic evaluation that should include 17-hydroxyprogesterone measurement plus assessment of ovulation and ovarian ultrasound. (Fertil Steril ${ }^{\circledR}$ 2010;94:194-7. C 2010 by American Society for Reproductive Medicine.)
\end{abstract}

Key Words: Hyperandrogenism, PCOS, hirsultism, nonclassic adrenal hyperplasia

Hirsutism is a common condition in which women present terminal hairs with a male-like pattern (1). In most patients, hirsutism is a sign of disorders that may produce significant morbidity (2). Therefore, treatment of hirsutism has to be preceded by a careful diagnostic evaluation, and the findings will largely influence the therapeutic decisions.

Recently, the Endocrine Society has published a clinical guideline on evaluation and treatment of hirsutism (3). This guideline, although useful and based on review of available evidence, presents some conclusions that contrast with the personal experience of the invesitgator and with that of many experts. In particular, the investigators stated that patients with mild hirsutism and no other important clinical signs (menstrual irregularities, infertility, central obesity, acanthosis nigricans, rapid progression of the hirsutism, clitoromegaly) should not be further studied, although they

Received December 15, 2008; revised February 11, 2009; accepted February 13, 2009; published online April 1, 2009.

G.D.F. has nothing to disclose. P.M. has nothing to disclose. I.P. has nothing to disclose. G.B.R. has nothing to disclose. E.C. has nothing to disclose.

Reprint requests: Enrico Carmina, M.D., Department of Clinical Medicine, University of Palermo, via delle Croci 47, 90139 Palermo, Italy (FAX: 390916552953; E-mail: enricocarmina@libero.it, enrico.carmina@ ae-society.org). acknowledged that this recommendation was based on lowquality evidence (3).

Of course, personal experiences cannot be considered when preparing guidelines based on available evidence. Therefore, in this study, we wished to verify what diagnosis has been performed during the last 10 years in our department in patients referred because of mild hirsutism who did not present the clinical signs indicated in the guideline of Endocrine Society.

\section{MATERIALS AND METHODS}

Between 1999 and 2008, 420 women were studied because of hirsutism in the Endocrine Unit of the Department of Clinical Medicine, at the University of Palermo.

Hirsutism was assessed by Ferriman-Gallwey-Lorenzo scores (4) (patients with eight or more scores were considered hirsute). Three hundred forty-four patients presented a mild hirsutism (8-15 FGL scores), whereas 76 had a moderate-severe hirsutism ( $>15$ FGL scores).

Only the records that contained at least the following studies were considered useful for inclusion of hirsute patients in this study: physical examination, body mass index (BMI), 
waist circumference (WC), age, and characters of menstrual cycles.

Physical examination showing medium-severe hirsutism ( $>15$ FGL scores), clitoromegaly, acanthosis nigricans, $\mathrm{BMI} \geq 30$ or WC $\geq 88 \mathrm{~cm}$ represented a criterion of exclusion. Menstrual cycles shorter than 25 days and longer than 34 days were considered abnormal, and represented a criterion of exclusion. History of rapid progression of hirsutism in the last few months represented another criterion of exclusion.

One hundred eighty women, presenting mild hirsutism, were excluded because of clinical features (BMI, WC, clitoromegaly, acantosis nigricans, abnormal menses), whereas 12 patients with mild hirsutism were excluded because of an incomplete diagnostic workup. One hundred fifty-two women, presenting mild hirsutism (8-15 FGL scores) and no criteria of exclusion, were included in this study. In these patients, serum levels of testosterone (T), dehydroepiandrosterone sulphate (DHEAS), 17-hydroxyprogesterone (17OHP), progesterone (P), and pelvic sonography were evaluated at the moment of their referral to our endocrine clinic.

Serum androgens and 17OHP were measured during follicular phase (days 5-8), whereas serum $\mathrm{P}$ was determined on days 21-24 of the menstrual cycle.

Serum hormone levels were quantified by well-established methods that had been validated previously in our laboratory. All steroids were measured by specific RIAs after extraction using previously described methods (5). In all assays, intraassay and interassay coefficients of variation did not exceed $6 \%$ and $15 \%$, respectively.

Biochemical hyperandrogenism was defined as serum $\mathrm{T}>60 \mathrm{ng} / \mathrm{dL}(>2.08 \mathrm{nmol} / \mathrm{L})$ and/or serum DHEAS $\geq 3,000 \mu \mathrm{g} / \mathrm{L}$ ( $\geq 7.8 \mu \mathrm{mol} / \mathrm{L})$. These values of hyperandrogenism have been previously calculated in our population with the same assays (6).

Increased serum 17OHP was defined as serum 17OHP $>3$ $\mu \mathrm{g} / \mathrm{L}(>9.1 \mathrm{nmol} / \mathrm{L})$. In patients with mildly increased serum 17OHP $(<10 \mu \mathrm{g} / \mathrm{L}$ and $>3 \mu \mathrm{g} / \mathrm{L})$, increased $17 \mathrm{OHP}$ response to ACTH administration ( $1 \mathrm{mg}$ IV with blood samples at 0 , 30 , and 60 minutes) was required for diagnosis of nonclassic 21hydroxylase deficiency (7).

Anovulation was defined as serum $\mathrm{P}<3 \mathrm{ng} / \mathrm{mL}(<9.54$ $\mathrm{nmol} / \mathrm{L}$ ). In patients with normal menses, at least two consecutive menstrual cycles were studied, and finding of low levels of serum $\mathrm{P}(<3 \mathrm{ng} / \mathrm{mL})$ in both cycles indicated the presence of chronic anovulation.

Pelvic ultrasound had to report ovarian size (by measurement of the main three ovarian diameters in both ovaries) and presence, size, and number of ovarian microcysts. Both data obtained by abdominal and transvaginal ultrasounds were considered useful for the diagnosis.

The presence of polycystic ovaries was established by the presence of 10 or more peripherally oriented cystic structures in one ultrasonographic plane, each of which measured 2 to
$10 \mathrm{~mm}$ in diameter, arranged around a dense stroma (8). Ovarian volume was calculated by the formula $\pi / 6\left(D_{1} \times\right.$ $D_{2} \times D_{3}$ ), where the dimensions $(D)$ of length, width, and thickness were used. The size of both ovaries was assessed and mean ovarian size was calculated. According to our data of normal women, increased ovarian size indicates that the mean ovarian size was larger than $7.5 \mathrm{cc}^{3}$ (9). Similar data have been reported by others (10).

The diagnosis of the different androgen excess disorders was performed according previously reported criteria (11). In particular, polycystic ovary syndrome (PCOS) was diagnosed in hirsute patients presenting chronic anovulation and/or polycystic ovaries $(12,13)$. Diagnoses of PCOS included both ovulatory (OV-PCOS) and anovulatory (classic PCOS, PCOS) form. Ovulatory PCOS was diagnosed in patients having normal ovulatory menses, hyperandrogenism (clinical finding of hirsutism was sufficient), and polycystic ovarian morphology (13). Classic PCOS was diagnosed in patients having chronic anovulation and hyperandrogenism (clinical finding of hirsutism was sufficient). Nonclassic 21-adrenal hyperplasia was diagnosed on the basis of increased serum $17 \mathrm{OHP}(>10 \mu \mathrm{g} / \mathrm{L})(>30.3 \mathrm{nmol} / \mathrm{L})$ or mildly increased serum 17OHP $(>3<10 \mu \mathrm{g} / \mathrm{L})$ with an increased response to ACTH $(\Delta 17 \mathrm{OHP}>5 \mu \mathrm{g} / \mathrm{L}$ with a $17 \mathrm{OHP}$ peak $>10$ $\mu \mathrm{g} / \mathrm{L})$. Non-PCOS hyperandrogenism was diagnosed in hirsute patients presenting ovulatory cycles and normal ovaries at the ultrasound study. According their androgen levels, these patients were further distinguished in idiopathic hyperandrogenism and idiopathic hirsutism (13).

Fifty normal nonhirsute controls were also studied. They were students or young doctors, matched for age and BMI, who volunteered for this study. All studied controls showed normal androgen levels, normal ovulatory cycles, and normal ovaries.

Institutional review board approval was obtained, and all patients and controls gave written consent. All subjects were considered to be sedentary, and were not dieting or receiving any medications. No subject received hormonal medications for at least 3 months before the study.

\section{Statistical Analysis}

A two-group comparison of continuous variables was performed using a two-sample $t$ test with adjustment for nonconstancy of variance, when necessary. A Bartlett test was used to evaluate homogeneity of variance. More than two group means were compared using analysis of variance with post hoc least-squares means pairwise comparisons. All data are presented as mean \pm standard deviation.

\section{RESULTS}

The mean clinical and hormonal data of 152 patients with mild hirsutism are presented in Table 1. Patients and controls were similar for age and BMI. However, hirsute patients presented significantly higher WC $(P<.01)$ FGL scores $(P<.01)$, 


\section{TABLE 1}

Clinical data and serum androgen values in 152 women with mild hirsutism (FGL scores 8 to 15) and 50 nonhirsute controls.

\begin{tabular}{|c|c|c|c|c|c|c|}
\hline & Age (y) & BMI & WC (cm) & FGL scores & $T$ (ng/dL) & DHEAS ( $\mu \mathrm{g} / \mathrm{L})$ \\
\hline $\begin{array}{l}\text { Patients with mild hirsutism } \\
\text { Controls } \\
\text { Normal range }\end{array}$ & $\begin{array}{l}24.2 \pm 5 \\
24.6 \pm 3\end{array}$ & $\begin{aligned} 22.7 & \pm 2.3 \\
22 & \pm 2 \\
& <25\end{aligned}$ & $\begin{aligned} 78 & \pm 4.9^{\mathrm{a}} \\
76 & \pm 4 \\
& <80\end{aligned}$ & $\begin{array}{c}9.9 \pm 2.5^{a} \\
3 \pm 1 \\
0-5\end{array}$ & $\begin{array}{c}64 \pm 28^{a} \\
34 \pm 13 \\
20-60\end{array}$ & $\begin{array}{l}2.6 \pm 1.2^{a} \\
2.1 \pm 0.4 \\
1.2-2.9\end{array}$ \\
\hline \multicolumn{7}{|c|}{$\begin{array}{l}\text { Note: } \mathrm{BMI}=\text { body mass index; } \mathrm{WC}=\text { waist circumference; } \mathrm{DHEAS}=\text { dehydroepiandrosterone sulfate. Mean } \pm 2 \mathrm{SD} \\
\text { values of controls was used to calculate the normal ranges of } \mathrm{FGL} \text { scores, } \mathrm{T} \text {, and DHEAS. Normal limits of BMI and } \\
\text { WC were derived by epidemiologic studies in the same population. } \\
{ }^{a} P<.01 \text { compared to controls. }\end{array}$} \\
\hline
\end{tabular}

serum T $(P<.01)$, and serum DHEAS $(P<.01)$ than control women.

The analysis of individual values showed that $86(57 \%)$ patients presented increased values of $\mathrm{T}$, whereas 37 (24.5\%) hirsute women had increased serum levels of DHEAS. Because most patients with increased DHEAS had also increased $\mathrm{T}$, the total number of hyperandrogenic women was 91 (59.9\% of the women with mild hirsutism). Seventy patients $(46 \%)$ with mild hirsutism had values of WC between 80 and $87 \mathrm{~cm}$.

Table 2 shows the distribution of different androgen excess disorders in the patients with mild hirsutism. Most patients $(80=52.6 \%)$ had a non-PCOS disorder that in 43 women were associated with increased androgen levels (idiopathic hyperandrogenism), whereas in 34 women $\mathrm{T}$ and DHEAS were normal (idiopathic hirsutism).

Three patients presented a nonclassic 21-hydroxylase deficiency (NCAH).

Polycystic ovary syndrome was found in 72 (47.4\%) women with mild hirsutism and no other clinical signs. Most PCOS women with mild hirsutism were ovulatory $(56=$ $36.8 \%$ ), but 16 were anovulatory (classic PCOS: $10.6 \%$ ).

Values of WC $<80 \mathrm{~cm}$ and between 80 and $87 \mathrm{~cm}$ did not distinguish between PCOS and non-PCOS patients.

\section{DISCUSSION}

The data of this retrospective study confirm the clinical experience that severity of hirsutism cannot be used to distinguish patients. The addition of clinical data such as normality of menses and normal BMI and WC, although increasing the probability of finding a non-PCOS disorder, does not rule out the possibility of a diagnosis of PCOS or nonclassic adrenal hyperplasia. In our study, half (47.4\%) of the patients with mild hirsutism and no other clinical signs had PCOS (according to Rotterdam or AES criteria) or nonclassic adrenal hyperplasia (2\%). In addition, whereas most patients with PCOS had the mild ovulatory form, 16 (10.6\%) women had the classic anovulatory phenotype that corresponds to previ- ous NIH diagnostic criteria for diagnosis of PCOS (14). On the other hand, it is well known that although in general population normal menses are generally associated to ovulatory cycles, in hyperandrogenic women anovulation may be present in a significant proportion of women with apparently normal cycles. Because of this, previous studies have found that the anovulatory form of PCOS may present in $10 \%$ to $20 \%$ of normally cycling women $(15,16)$.

Although this was a retrospective study, we do not believe it represents a motive of bias. In fact, during last 10 years, in our department, all patients with hirsutism did a similar initial study, independently on severity of hirsutism. Of course, a prospective study may be needed to confirm our experience.

It is important to observe that most of the hirsute patients referred to our department during the last 10 years presented a mild hirsutism. In fact, out of 420 hirsute patients, 344 patients presented a mild hirsutism (8-15 FGL scores), whereas 76 had a moderate-severe hirsutism ( $>15$ FGL scores). Therefore, mild hirsutism is present in most hirsute patients (82\%) and exclusion of these patients from a careful study may restrain from performing the diagnosis of PCOS in a significant number of patients.

Because prevalence of PCOS (National Institutes of Health defined) in the general female population is around 6\% (17), our findings show that the presence of mild hirsutism, without

\section{TABLE 2}

Diagnosis in 152 women with mild hirsutism.

Patients PCOS OV-PCOS NCAH No PCOS

$\begin{array}{lllll}\text { Number } & 16 & 56 & 3 & 77 \\ \% & 10.6 & 36.8 & 2 & 50.6\end{array}$

Note: PCOS = polycyctic ovary syndrome; OV-PCOS = ovulatory form PCOS; $\mathrm{NCAH}=$ nonclassic 21-hydroxylase deficiency.

${ }^{a}$ No PCOS indicates hirsute patients where no diagnosis of PCOS or NCAH was performed.

Di Fede. PCOS in mildly hirsute women. Fertil Steril 2010 
abdominal obesity or anovulation, increases only slightly (about 1.8 times) the PCOS prevalence (at least of more severe phenotype) over the general population. However, it has to be remembered that this is just a subgroup of hirsute patients. Most patients with mild hirsutism were excluded by this study because of obesity (at least abdominal obesity) and/or anovulation and, whereas in these patients the prevalence of classic PCOS was not assessed, it is probable that anovulatory PCOS is more common in patients with more severe phenotype. Similarly, prevalence of classic PCOS may be higher in patients with severe hirsutism. In addition, the prevalence of the milder ovulatory form was highly increased, and also this PCOS phenotype is able to determine a higher metabolic and cardiovascular risk (18).

Our data indicate that the conclusions of the recent Endocrine Society guideline, that stated that patients with mild hirsutism should not be further investigated, cannot be accepted. In our view, it is just the opposite. In patients with hirsutism and menstrual irregularities the diagnosis of PCOS does not require further data, and although it may be useful to have androgen levels and ovarian ultrasound study, all it does not modify the diagnosis or the treatment of the patients. Probably, in these women, it is more important to evaluate the metabolic pattern (serum lipids, oral glucose tolerance test, insulin resistance) to understand what other problems these patients may have.

On the contrary, the patients with mild hirsutism and no clinical signs require further investigation because the possible diagnoses are completely different and may require different treatments or different need of follow-up. Because of this, we suggest that all patients with mild hirsutism and no clinical signs should perform an ovarian sonography, an assessment of ovulation, and the measurement of serum 17OHP. Although we continue doing our reliable noncommercial androgen assays for research purposes, in clinical practice, especially in patients with mild hirsutism and no other clinical signs, there is no need to evaluate serum androgens because clinical hyperandrogenism is already present and commercial androgen assays are of little value.

\section{REFERENCES}

1. Carmina E, Lobo RA. Hirsutism, alopecia and acne. In: Beckers KL, ed. Principles and practice of endocrinology and metabolism. 3rd ed. Philadelphia, PA: Lippincott Publishing, 2001:991-1008.
2. Carmina E, Lobo RA. Polycystic ovary syndrome (PCOS), arguably the most common endocrinopathy, is associated with significant morbidity in women. J Clin Endocrinol Metab 1999;84:1897-9.

3. Martyn KA, Chang RJ, Ehrmann DA, Ibanez L, Lobo RA, Rosenfield RL, et al. Evaluation and treatment of hirsutism in premenopausal women: an Endocrine Society Clinical Practice Guideline. J Clin Endocrinol Metab 2008;93:1105-20.

4. Hatch R, Rosenfield RL, Kim MH, Tredway D. Hirsutism: implications, etiology and management. Am J Obstet Gynecol 1981;140:815-30.

5. Carmina E, Stanczyk F, Chang L, Miles RA, Lobo RA. The ratio of

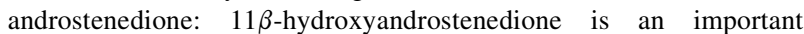
marker of adrenal androgen excess in women. Fertil Steril 1992;58: $148-52$.

6. Carmina E. Prevalence of idiopathic hirsutism. Eur J Endocrinol 1998;139:421-3.

7. Carmina E, Lobo RA. Ovarian suppression reduces clinical and endocrine expression of late onset congenital adrenal hyperplasia due to 21hydroxylase deficiency. Fertil Steril 1994;62:738-43.

8. Balen AH, Laven JS, Tan SL, Dewailly D. Ultrasound assessment of the polycystic ovary: international consensus definitions. Hum Reprod Update 2003;9:505-14.

9. Carmina E, Orio F, Palomba S, Longo RA, Lombardi G, Lobo RA. Ovarian size and blood flow in women with polycystic ovary syndrome (PCOS) and their correlations with some endocrine parameters. Fertil Steril 2005;84:413-9.

10. Jonard S, Robert Y, Dewailly D. Revisiting the ovarian volume as a diagnostic criterion for polycystic ovaries. Hum Reprod 2005;20: 2893-8.

11. Carmina E, Rosato F, Jannì A, Rizzo M, Longo RA. Relative prevalence of different androgen excess disorders in 950 women referred because of clinical hyperandrogenism. J Clin Endocrinol Metab 2006;91:2-6.

12. Azziz R, Carmina E, Dewailly D, Diamanti-Kandarakis E, EscobarMorreale HF, Futterweit W, et al. Position statement: criteria for defining polycystic ovary syndrome as a predominantly hyperandrogenic syndrome: an androgen excess society guideline. J Clin Endocrinol Metab 2006;91:4237-45.

13. Carmina E. The spectrum of androgen excess disorders. Fertil Steril 2006;85:1582-5.

14. Zawdaki JK, Dunaif A. Diagnostic criteria for polycystic ovary syndrome: towards a rationale approach. In: Dunaif A, Givens JR, Haseltine F, Merriam GR, eds. Polycystic ovary syndrome. Boston, MA: Blackwell Scientific Publications, 1992:377-84.

15. Azziz R, Waggoner WT, Ochoa T, Knochenauer ES, Boots LR. Idiopathic hirsutism: an uncommon cause of hirsutism in Alabama. Fertil Steril 1998;70:274-8.

16. Carmina E, Lobo RA. Do hyperandrogenic women with normal menses have polycystic ovary syndrome? Fertil Steril 1999;71:319-22.

17. Knochenhauer ES, Key TJ, Kahsar-Miller W, Waggoner W, Boots LR, Azziz R. Prevalence of the polycystic ovary syndrome in unselected black and white women of the South Eastern USA: a prospective study. J Clin Endocrinol Metab 1998;83:3078-82.

18. Carmina E, Longo RA, Rini GB, Lobo RA. Phenotypic variation in hyperandrogenic women influences the finding of abnormal metabolic and cardiovascular risk parameters. J Clin Endocrinol Metab 2005;90: 2545-9. 